\title{
CHARACTERIZING ALGEBRAIC STACKS
}

\author{
SHARON HOLLANDER
}

(Communicated by Paul Goerss)

\begin{abstract}
We extend the notion of algebraic stack to an arbitrary subcanonical site $\mathcal{C}$. If the topology on $\mathcal{C}$ is local on the target and satisfies descent for morphisms, we show that algebraic stacks are precisely those which are weakly equivalent to representable presheaves of groupoids whose domain map is a cover. This leads naturally to a definition of algebraic $n$-stacks. We also compare different sites naturally associated to a stack.
\end{abstract}

\section{INTRODUCTION}

Stacks arise naturally in the study of moduli problems in geometry. They were introduced by Giraud [Gi] and Grothendieck and were used by Deligne and Mumford $[\mathrm{DM}]$ to study the moduli spaces of curves. They have recently become important also in differential geometry [Bry] and homotopy theory [G]. Higher-order generalizations of stacks are also receiving much attention from algebraic geometers and homotopy theorists.

In this paper, we continue the study of stacks from the point of view of homotopy theory started in $[\mathrm{H}, \mathrm{H} 2]$. The aim of these papers is to show that many properties of stacks and classical constructions with stacks are homotopy theoretic in nature. This homotopy-theoretical understanding gives rise to a simpler and more powerful general theory. In $[\mathrm{H}]$ we introduced model category structures on different ambient categories in which stacks are the fibrant objects and showed that they are all Quillen equivalent. In this paper we work with the simplest such model: the local model structure on presheaves of groupoids on site $\mathcal{C}$, which we denote by $P(\mathcal{C}, \mathcal{G r p d})_{L}$.

Deligne and Mumford introduced the notion of an algebraic stack in [DM, Definition 4.6]. This definition generalizes easily to an arbitrary site $\mathcal{C}$, and our main result is a characterization of these (generalized) algebraic stacks on sites satisfying certain mild hypotheses.

A key observation is that the (2-category) fiber product (see Definition 2.1) is a model for the homotopy pullback in the model category $P(\mathcal{C}, \mathcal{G r p d})_{L}$, and this allows us to rewrite the definition of representable morphism in the following homotopy theoretic fashion:

$$
f: \mathcal{M} \rightarrow \mathcal{N} \in P(\mathcal{C}, \mathcal{G r p d})
$$

is representable if for each $X \rightarrow \mathcal{N}$ with $X \in \mathcal{C}$, the homotopy pullback $\mathcal{M} \times \mathcal{N}_{\mathcal{N}}^{h} X$ is weakly equivalent to a representable functor.

Received by the editors May 30, 2006 and, in revised form, June 29, 2006.

2000 Mathematics Subject Classification. Primary 55U10; Secondary 18G55, 14A20. 
Generalizing [DM, Definition 4.4], we say that a presheaf of groupoids $\mathcal{M}$ on $\mathcal{C}$ is algebraic if the diagonal $\mathcal{M} \rightarrow \mathcal{M} \times \mathcal{M}$ is a representable morphism and there exists a cover $X \rightarrow \mathcal{M}$ with $X \in \mathcal{C}$. By a cover we mean a representable morphism such that for all $Y \in \mathcal{C}$, the homotopy pullback

$$
X \times{ }_{\mathcal{M}}^{h} Y \rightarrow Y
$$

is weakly equivalent to a cover in $\mathcal{C}$.

We say that the (basis for the) topology on $\mathcal{C}$ is local if the notion of cover is local on the target (Definition 4.2). This condition is satisfied by virtually all the topologies in use in algebraic geometry, and one can always saturate a basis for a topology so that this condition is satisfied. A topology on $\mathcal{C}$ satisfies descent for morphisms if the contravariant assignment $X \mapsto \operatorname{Iso}(\mathcal{C} / X)$ is a stack (Definition 4.1). Our main result is then the following.

Theorem 1.1 (Theorem 4.3). Let $\mathrm{C}$ be a Grothendieck topology that is local on the target and satisfies descent for morphisms. $\mathcal{N} \in P(\mathcal{C}, \mathcal{G r p d})$ is algebraic if and only if $\mathcal{M}$ is weakly equivalent in $P(\mathcal{C}, \mathcal{G r p d})_{L}$ to a representable presheaf of groupoids $\left(X_{o}, X_{m}\right)$ with the domain map $X_{m} \rightarrow X_{o}$ a cover in $\mathcal{C}$.

In particular, if $\mathcal{C}=\mathrm{Aff}_{\text {flat }}$ (fpqc for affine schemes), then this theorem characterizes algebraic stacks as those weakly equivalent to flat Hopf algebroids.

This result leads naturally to a definition of algebraic $\infty$-stacks ( $n$-stacks); they are those presheaves of simplicial sets on $\mathcal{C}$ that are weakly equivalent in $P(\mathcal{C}, s \mathcal{S} e t)_{L}$ (see $[\mathrm{DHI}])$ to (the $n$-coskeleton of) a simplicial object in $\mathcal{C}$ where all the boundary maps are covers.

In the appendix we consider several natural sites associated to a stack and compare them. The first is the classical site $\mathcal{C} / \mathcal{M}$ (see $[D M]$ ). In this topology, the objects are maps $X \rightarrow \mathcal{M}$ with $X \in \mathcal{C}$. It is natural to ask for a topology on the over category of $\mathcal{M}$ (in which $\mathcal{M}$ itself is an object). We use the notion of representability to construct a larger site $\operatorname{Rep} / \mathcal{M}$ and prove that for $\mathcal{M}$ algebraic the sheaves on the two sites agree.

We also construct a topology on $P(\mathcal{C}, \mathcal{G r p d}) / \mathcal{M}$ where the covers are collections of fibrations $\mathcal{U}_{i} \rightarrow \mathcal{M}$ such that the canonical map from the realization of the nerve $\left|\mathcal{U}_{\bullet}\right| \rightarrow \mathcal{M}$ is a weak equivalence. We characterize these covers as those sets of maps whose image is locally a covering sieve for the topology on $\mathcal{C}$ (see Proposition A.9).

1.1. Relation to other work. In his 2004 Northwestern thesis E. Pribble [P] constructs an equivalence of 2-categories between flat Hopf algebroids and rigidified algebraic stacks. This is essentially equivalent to Theorem 4.3 in case $\mathcal{C}$ is affine schemes in the flat topology.

1.2. Acknowledgments. I would like to thank G. Granja for helpful comments.

1.3. Notation and conventions. We assume that our fixed base site $\mathcal{C}$ is small and closed under finite products and pullbacks. By topology we mean what is usually called a basis for a topology [MM, Definition III.2.2]. We assume the topology is subcanonical, i.e., that the representable functors are sheaves, and identify the objects in $\mathcal{C}$ with the sheaves they represent.

We write $P(\mathcal{C}, \mathcal{G r p d})$ for the category of presheaves of groupoids on $\mathcal{C}$. If $\left\{U_{i} \rightarrow\right.$ $X\}$ is a cover, we write $U=\coprod_{i} U_{i}$ for the coproduct of the sheaves and $U$ • for the nerve of the cover, which is the simplicial object obtained by taking iterated fiber 
products over $X$. We will sometimes abuse notation and write a cover as $U \rightarrow X$. $\left|U_{\bullet}\right|$ will denote the geometric realization of the simplicial object in $P(\mathcal{C}, \mathcal{G r p d})$. Recall that the geometric realization of a simplicial diagram $F_{\bullet}$ in $P(\mathcal{C}, \mathcal{G r p d})$ is defined by $\left|F_{\bullet}\right|(X)=\left|F_{\bullet}(X)\right|$ (see $[$ H, Section 2.2]).

We will write $P(\mathcal{C}, \mathcal{G r p d})$ for the category of presheaves of groupoids with the levelwise model structure where a map $F \rightarrow F^{\prime}$ is a fibration (weak equivalence) if and only if $F(X) \rightarrow F^{\prime}(X)$ is a fibration (weak equivalence) in $\operatorname{Grpd}$ for all $X \in \mathrm{C}$. We will write $P(\mathcal{C}, \mathcal{G r p d})_{L}$ for the local model structure, which is the localization of $P(\mathcal{C}, \mathcal{G r p d})$ with respect to the maps $\left|U_{\bullet}\right| \rightarrow X$, where $U \rightarrow X$ is a cover (see $[\mathrm{H}]$ ). The local model structure $P(\mathcal{C}, \mathcal{G r p d})_{L}$ is our default.

We will use repeatedly the basic result $[H$, Theorem 5.7] which characterizes the weak equivalences in $P(\mathrm{C}, \mathcal{G r p d})_{L}$ as those satisfying the local lifting conditions.

Definition 1.2 ([H, Definition 5.6]). A map $F \rightarrow G \in P(\mathcal{C}, \mathcal{G} r p d)$ satisfies the local lifting conditions if

(1) Given a commutative square
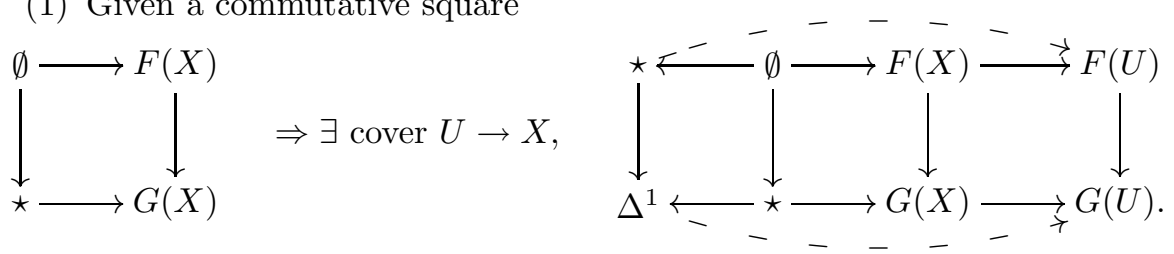

(2) For $A \rightarrow B$, one of the generating cofibrations $\partial \Delta^{1} \rightarrow \Delta^{1}, B \mathbb{Z} \rightarrow \star$, given a commutative square
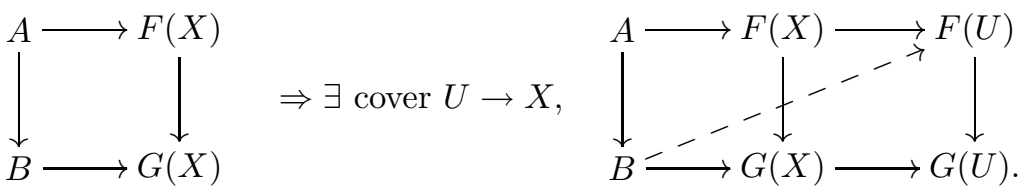

\section{Fiber PROduct}

In this section we will review the fiber product of stacks [LM-B, Definition 2.2.2] from our homotopy-theoretic point of view.

Definition 2.1. Let

$$
\mathcal{M}_{1} \stackrel{i}{\rightarrow} \mathcal{N} \stackrel{j}{\leftarrow} \mathcal{M}_{2}
$$

be a diagram in $P(\mathcal{C}, \mathcal{G r p d})$. The homotopy fiber product $\mathcal{M}_{1} \times{ }_{\mathcal{N}}^{h} \mathcal{M}_{2}$ is the presheaf of groupoids defined as follows:

(1) the objects of $\left(\mathcal{M}_{1} \times{ }_{\mathcal{N}}^{h} \mathcal{M}_{2}\right)(X)$ are triples $(a, b, \phi)$ with $a \in \mathcal{M}_{1}(X), b \in$ $\mathcal{M}_{2}(X)$ and an isomorphism $\phi: i(a) \stackrel{\sim}{\longrightarrow} j(b)$, and

(2) morphisms of $\left(\mathcal{M}_{1} \times{ }_{\mathcal{N}}^{h} \mathcal{M}_{2}\right)(X)$ from $(a, b, \phi)$ to $\left(a^{\prime}, b^{\prime}, \phi^{\prime}\right)$ are pairs $(\alpha, \beta)$, where $\alpha: a \cong a^{\prime}$ and $\beta: b \cong b^{\prime}$, such that $\phi^{\prime} \circ i(\alpha)=j(\beta) \circ \phi$.

There are natural projections $p_{i}: \mathcal{M}_{1} \times \mathcal{N}_{\mathcal{N}}^{h} \mathcal{M}_{2} \rightarrow \mathcal{M}_{i}$ and a natural homotopy $i \circ p_{1} \rightarrow j \circ p_{2}$ which are universal in the following sense. To give a map $f: \mathcal{M} \rightarrow$ $\mathcal{M}_{1} \times{ }_{\mathcal{N}}^{h} \mathcal{M}_{2}$ is the same as to give maps $f_{i}: \mathcal{M} \rightarrow \mathcal{M}_{i}$ and a levelwise homotopy $i \circ f_{1} \rightarrow j \circ f_{2}$.

The homotopy fiber product defined above is obviously the homotopy limit of the pullback diagram in the category $P(\mathcal{C}, \mathcal{G r p d})$ with the levelwise model structure. In 
fact, it also provides a model for the homotopy pullback in the local model structure as we now see.

Lemma 2.2. The homotopy fiber product of Definition 2.1 is a model for the homotopy pullback in $\mathrm{P}(\mathrm{C}, \mathcal{G} r p d)_{L}$.

Proof. Consider the pullback diagram in Definition 2.1. Since $P(\mathcal{C}, \mathcal{G r p d})_{L}$ is right proper [H, Corollary 5.8] (and $P(\mathcal{C}, \mathcal{G} r p d$ ) is obviously right proper), the homotopy fiber product in both of these model categories is obtained by replacing the map $\mathcal{M}_{2} \rightarrow \mathcal{N}$ by a fibration and taking the pullback.

Factor $\mathcal{M}_{2} \rightarrow \mathcal{N}$ into a trivial cofibration followed by a fibration $\mathcal{M}_{2} \stackrel{\sim}{\longrightarrow} \mathcal{M}^{\prime} \rightarrow \mathcal{N}$ in $P(\mathcal{C}, \mathcal{G r p d})_{L}$. Further factor $\mathcal{M}_{2} \stackrel{\sim}{\longrightarrow} \mathcal{M}^{\prime}$ into a levelwise trivial cofibration and a levelwise fibration $\mathcal{M}_{2} \stackrel{\sim}{\longrightarrow} \mathcal{M}^{\prime \prime} \rightarrow \mathcal{M}^{\prime}$. Then we have a levelwise weak equivalence

$$
\mathcal{M}_{1} \times{ }_{\mathcal{N}}^{h} \mathcal{M}_{2} \simeq \mathcal{M}_{1} \times \mathcal{N} \mathcal{M}^{\prime \prime}
$$

The map $\mathcal{M}_{1} \times \mathcal{N} \mathcal{M}^{\prime \prime} \rightarrow \mathcal{M}_{1} \times \mathcal{N} \mathcal{M}^{\prime}$ is the pullback of a levelwise fibration and weak equivalence and hence, by [H, Corollary 5.8], it is itself a weak equivalence.

Remark 2.3. Since homotopy limits commute with each other, if $\mathcal{M}_{1}, \mathcal{M}_{2}$ and $\mathcal{N}$ in Definition 2.1 are stacks (presheaves of groupoids satisfying the homotopy sheaf condition; see [H, Definition 1.3]), then the homotopy fiber product $\mathcal{M}_{1} \times{ }_{\mathcal{N}}^{h} \mathcal{M}_{2}$ is also a stack and agrees with what is usually called the fiber product of stacks [LM-B, 2.2.2].

Given a groupoid object $\left(X_{0}, X_{1}\right)$ in $\mathcal{C}$ we abuse notation and let $\left(X_{0}, X_{1}\right)$ denote the presheaf of groupoids of which $X_{0}$ represents the objects and $X_{m}$ represents the morphisms. We let $\mathcal{M}_{\left(X_{0}, X_{1}\right)}$ denote the fibrant replacement in $P(\mathcal{C}, \mathcal{G r p d})_{L}$ of $\left(X_{0}, X_{1}\right)$, that is, its stackification.

Lemma 2.4. Let $\mathcal{M}$ be a presheaf of groupoids, $\left(X_{0}, X_{1}\right)$ be a groupoid object in $\mathrm{C}$, and $\left(X_{0}, X_{1}\right) \rightarrow \mathcal{M}$ a weak equivalence in $P(\mathcal{C}, \mathcal{G r p d})_{L}$. The map

$$
X_{1} \longrightarrow X_{0} \times{ }_{\mathcal{M}}^{h} X_{0}
$$

induced by the domain and range is a weak equivalence. If $\mathcal{M}$ is a stack, it is a levelwise weak equivalence.

Proof. First we prove that the map is a weak equivalence for $\mathcal{M}=\mathcal{M}_{\left(X_{0}, X_{1}\right)}$. By Lemma 2.2, we need to verify the local lifting conditions of Definition 1.2 for the map $X_{1} \rightarrow X_{0} \times{ }_{\mathcal{M}}^{h} X_{0}$. By definition the map $\left(X_{0}, X_{1}\right) \rightarrow \mathcal{M}$ is a weak equivalence and so by 1.2(2), given two objects $a, b \in X_{0}(Y)$ and an isomorphism between their images in $\mathcal{M}(Y)$, there exists a cover $U \rightarrow Y \in \mathcal{C}$ such that this isomorphism lifts to $X_{1}(U)$. This implies that condition 1.2(1) holds for the map $X_{1} \rightarrow X_{0} \times{ }_{\mathcal{M}}^{h} X_{0}$.

Similarly, given $\phi_{1}, \phi_{2} \in X_{1}(X)$, an isomorphism between their images are in $\left(X_{0} \times{ }_{\mathcal{M}}^{h} X_{0}\right)(X)$ is necessarily trivial (as $X_{0}(X)$ is discrete) and so the images of $\phi_{1}$ and $\phi_{2}$ in $\mathcal{M}(X)$ are the same.

The fact that $\left(X_{0}, X_{1}\right) \rightarrow \mathcal{M}$ satisfies condition $1.2(2)$ for the cofibration $B \mathbb{Z} \rightarrow *$ guarantees the existence of the cover $U$ of $X$ such that $\phi_{1}$ and $\phi_{2}$ are in $X_{1}(U)$. This proves one half of $1.2(2)$ for the map $X_{1} \rightarrow X_{0} \times_{\mathcal{M}}^{h} X_{0}$, and the other half is automatic as $X_{1}(X)$ is discrete.

For general $\mathcal{M}$, the fact that the map is a weak equivalence follows from the homotopy invariance of the homotopy fiber product (Lemma 2.2). 
Since weak equivalences between fibrant objects in $P(\mathcal{C}, \mathcal{G r p d})_{L}$ are levelwise weak equivalences, if $\mathcal{M}$ is a stack, the map is a levelwise weak equivalence.

Remark 2.5. If $\mathcal{M}$ is a stack, the statement that $X_{1} \rightarrow X_{0} \times{ }_{\mathcal{M}}^{h} X_{0}$ is a levelwise weak equivalence means that, evaluating at each $X \in \mathcal{C}$,

$$
\left(X_{0}, X_{1}\right)(X) \rightarrow \mathcal{M}(X)
$$

is bijective on Hom sets and that two objects with the same image in $\mathcal{N}(X)$ are already isomorphic on $\left(X_{0}, X_{1}\right)(X)$. Thus this map is equivalent to the inclusion of a full subcategory of $\mathcal{M}(X)$ for each $X \in \mathcal{C}$.

If $\mathcal{M}$ is not a stack, composing the map with a fibrant replacement for $\mathcal{M}$ shows that $\left(X_{0}, X_{1}\right)(X) \rightarrow \mathcal{M}(X)$ is injective on morphisms and isomorphism classes.

\section{RePRESENTABLE MORPHISMS}

We begin by giving a definition of a representable morphism in $P(\mathcal{C}, \mathcal{G r p d})$ generalizing the one for stacks in [DM, Definition 4.2]. ${ }^{1}$ Classically the definition of representable morphism applies only to maps between stacks, for which the following two notions agree (by Lemma 2.2).

Definition 3.1. A morphism $\mathcal{M} \rightarrow \mathcal{N} \in P(\mathcal{C}, \mathcal{G} r p d)$ is called

- strongly representable if for each $X \in \mathcal{C}$ and each map $X \rightarrow \mathcal{N}$, the homotopy fiber product $X \times{ }_{\mathcal{N}}^{h} \mathcal{M}$ is levelwise weakly equivalent to a representable presheaf;

- representable if for each $X \in \mathcal{C}$ and each map $X \rightarrow \mathcal{N}$, the homotopy fiber product $X \times{ }_{\mathcal{N}}^{h} \mathcal{M}$ is weakly equivalent to a representable presheaf.

Representability allows one to extend certain properties of morphisms in $\mathcal{C}$ to arbitrary presheaves of groupoids.

Definition 3.2. Let $\mathrm{P}$ be a property of morphisms in $\mathrm{C}$. We say that $f: \mathcal{M} \rightarrow \mathcal{N}$ satisfies property $\mathrm{P}$ if for all maps $X \rightarrow \mathcal{N}$ with $X \in \mathcal{C}$, the map $X \times{ }_{\mathcal{N}}^{h} \mathcal{M} \rightarrow X$ is weakly equivalent to a map in $\mathcal{C}$ that satisfies property $\mathrm{P}$.

Similarly, a collection $\left\{\mathcal{U}_{i} \rightarrow \mathcal{N}\right\}$ is a cover if for each $X \rightarrow \mathcal{M}$ with $X \in \mathcal{C}$, $\left\{\mathcal{U}_{i} \times_{\mathcal{N}}^{h} X \rightarrow X\right\}$ is weakly equivalent to a cover in $\mathcal{C}$.

Notice that if $f$ satisfies property $\mathrm{P}$ as above, then it is necessarily representable.

Given a presheaf of groupoids $F$ recall that $\pi_{0} F$ is the presheaf of groupoids defined by $\left(\pi_{0} F\right)(X)=\pi_{0}(F(X))$.

Proposition 3.3. A map $f: \mathcal{M} \rightarrow \mathcal{N} \in P(\mathcal{C}, \mathcal{G r p d})$ is representable iff any fibration $p: \mathcal{M}^{\prime} \rightarrow \mathcal{N}^{\prime}$ weakly equivalent to $f$ is strongly representable. In that case, for each map $X \rightarrow \mathcal{M}$, (the sheaf) $\pi_{0}\left(X \times \mathcal{M} \mathcal{M}^{\prime}\right)$ is isomorphic to a representable functor.

Proof. If $p$ is strongly representable, $f$ is obviously representable. For the converse note that since $p$ is a fibration, given $X \rightarrow \mathcal{N}^{\prime}, X \times \mathcal{N}^{\prime} \mathcal{M}^{\prime}$ is levelwise weakly equivalent to the homotopy fiber product $X \times{ }_{\mathcal{N}^{\prime}}^{h} \mathcal{M}^{\prime}$, which is by assumption weakly equivalent to a representable functor. Now $X \times{ }_{\mathcal{N}^{\prime}} \mathcal{M}^{\prime} \rightarrow X$ is a fibration, representable functors are fibrant and weak equivalences between fibrant objects are levelwise weak equivalences. Hence $X \times{ }_{\mathcal{N}}^{h} \mathcal{M}$ is levelwise weakly equivalent to a representable functor.

The second statement is clear.

\footnotetext{
${ }^{1}$ In $[\mathrm{LM}-\mathrm{B}, 3.9]$ for $\mathcal{C}=$ Aff étale such morphisms are called schematic.
} 
Note 3.4. The previous lemma shows that a map $f: \mathcal{N} \rightarrow \mathcal{N}$ is representable iff the associated map of stacks is strongly representable.

3.1. Generalized algebraic stacks. In this section we define the concept of a generalized algebraic presheaf of groupoids. We first recall the definition of algebraic stack which appears in [DM, 4.6]. This is usually called a Deligne-Mumford stack and we follow suit.

Definition 3.5. Let $S$ be a scheme and let $\mathcal{C}$ be the category of $S$-schemes in the étale topology. A stack $\mathcal{M}$ is called a Deligne-Mumford stack if the diagonal $\mathcal{M} \rightarrow \mathcal{M} \times \mathcal{M}$ is representable, separated and quasi-compact and if it admits an étale cover $X \rightarrow \mathcal{M}$ with $X \in \mathcal{C}$.

Definition 3.6. Let $\mathcal{C}$ be a site. We say that $\mathcal{M} \in P(\mathcal{C}, \mathcal{G} r p d)$ is generalized algebraic if its diagonal is representable and there is a cover $X \rightarrow \mathcal{M}$ with $X \in \mathcal{C}$.

Note 3.7. The condition that the diagonal of $\mathcal{M}$ be representable is equivalent to the requirement that for all $X \rightarrow \mathcal{M}, Y \rightarrow \mathcal{M}$, with $X, Y \in \mathcal{C}$ the product $X \times_{\mathcal{M}}^{h} Y$ is weakly equivalent to a representable functor.

\section{Characterization of the Generalized Algebraic Stacks}

In this section we give a homotopy-theoretic characterization of generalized algebraic stacks (Theorem 4.3). For this we will need the following definition, which generalizes faithfully flat descent of morphisms [SGA, Theorem VIII.2.1].

Definition 4.1. Given a site $\mathcal{C}$ consider the presheaf of groupoids on $\mathcal{C}$ defined on objects by $X \mapsto i s o(\mathcal{C} / X)$ and on morphisms via pullback. We say that the site $\mathcal{C}$ satisfies descent for morphisms if this is a stack.

Definition 4.2. We say that a topology on $\mathcal{C}$ is local if the notion of cover is local on the target. This means that if $\left\{U_{i} \rightarrow X\right\}$ is a cover and $\left\{V_{j} \rightarrow X\right\}$ is a collection of morphisms such that $\left\{V_{j} \times_{X} U_{i} \rightarrow U_{i}\right\}$ is a cover for each $i$, then $\left\{V_{k} \rightarrow X\right\}$ is also a cover.

Theorem 4.3. Let $\mathcal{C}$ be a site that is local and satisfies descent for morphisms. Then $\mathcal{M}$ is a generalized algebraic presheaf of groupoids if and only if $\mathcal{M}$ is weakly equivalent in $P(\mathcal{C}, \mathcal{G r p d})_{L}$ to a groupoid object $\left(X_{o}, X_{m}\right)$ in $\mathcal{C}$, for which the domain map $X_{m} \rightarrow X_{o}$ is a cover.

The proof is broken down into the following two propositions.

Proposition 4.4. Let $\mathcal{M}$ be a generalized algebraic presheaf of groupoids and $X \rightarrow$ $\mathcal{M}$ be a cover (in the sense of Definition 3.2) with $X \in \mathcal{C}$. Let $X_{m}$ denote the representable functor weakly equivalent to $X \times_{\mathcal{M}}^{h} X$. Then the pair $\left(X, X_{m}\right)$ is a groupoid object in $\mathcal{C}$ and the natural map $\left(X, X_{m}\right) \rightarrow \mathcal{M}$ is a weak equivalence.

Proof. It suffices to prove this for $\mathcal{M}$ a stack. Given a generalized algebraic presheaf of groupoids there exists a representable morphism $X \rightarrow \mathcal{M}$ which is a cover. Let $X \stackrel{\sim}{\longrightarrow} \tilde{X} \rightarrow \mathcal{M}$ be a factorization as a trivial cofibration followed by a fibration, and let $\tilde{X}_{\bullet}$ denote the nerve of this cover.

$\tilde{X} \times \mathcal{M} X$ is levelwise weakly equivalent to a representable $X_{m}$, and $X_{m} \rightarrow X$ is a cover. Since $\left(\tilde{X}, \tilde{X} \times_{\mathcal{M}} \tilde{X}\right)$ is a groupoid object in the homotopy category, so is $\left(X, X_{m}\right)$, and as $X$ and $X_{m}$ are both fibrant, cofibrant, and discrete, $\left(X, X_{m}\right)$ is also a groupoid object in $P(\mathcal{C}, \mathcal{G} r p d)$ and in $\mathcal{C}$. 
Next we show that the map $\left(X, X_{m}\right)=\left|\left(X, X_{m}\right) \bullet\right| \rightarrow \mathcal{M}$ is a weak equivalence by verifying that it satisfies the local lifting conditions. The first of the local lifting conditions follows from the fact that $X \rightarrow \mathcal{M}$ is a cover. By Remark 2.5 the map $\left(X, X_{m}\right) \rightarrow \mathcal{M}$ is levelwise equivalent to the inclusion of a full subcategory and so the second of the local lifting conditions is also satisfied (even not locally).

Proposition 4.5. Let $\mathcal{C}$ be a site that is local and satisfies descent for morphisms. If $\left(X_{o}, X_{m}\right)$ is a groupoid object in $\mathcal{C}$, with $X_{m} \rightarrow X_{o}$ a cover, then the associated stack $\mathcal{M}_{\left(X_{o}, X_{m}\right)}$ is generalized algebraic.

Proof. First we will show that under these hypotheses, $X_{o} \rightarrow \mathcal{M}=\mathcal{M}_{\left(X_{o}, X_{m}\right)}$ is representable.

Let $Y \in \mathcal{C}$ and $Y \rightarrow \mathcal{M}$ be a map in $P(\mathcal{C}, \mathcal{G r p d})$. Since $X_{o} \rightarrow \mathcal{M}$ is locally surjective there is a cover $U \rightarrow Y$ for which we have the following factorization.

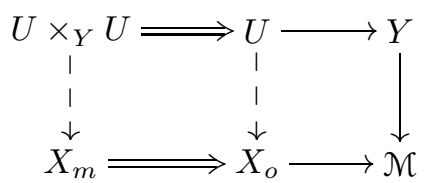

By construction of the homotopy fiber product we obtain a simplicial diagram of fibrations $U_{\bullet} \times{ }_{\mathcal{M}}^{h} X_{O} \rightarrow U$ • augmented by $Y \times{ }_{\mathcal{M}}^{h} X_{0} \rightarrow Y$. Notice that besides $Y \times{ }_{\mathcal{M}}^{h} X_{o}$ all of the fiber products $\left(U \times_{Y} U \cdots \times Y_{U}\right) \times_{\mathcal{M}}^{h} X_{O}$ are levelwise weakly equivalent to representable functors; for example,

$$
U \times_{\mathcal{M}}^{h} X_{o}=U \times_{X_{o}} X_{o} \times{ }_{\mathcal{M}}^{h} X_{o} \stackrel{\sim}{\longrightarrow} U \times_{X_{o}} X_{m} .
$$

Fix $V \in \mathcal{C}$ and $\alpha: U \times{ }_{\mathcal{M}}^{h} X_{o} \stackrel{\sim}{\longrightarrow} V$, and let $\bar{\alpha}$ be the induced isomorphism $\pi_{0}\left(U \times_{\mathcal{M}}^{h} X_{o}\right) \stackrel{\cong}{\longrightarrow} V$. Let $p r_{1}, p r_{2}$ denote the projections $U \times_{Y} U \rightarrow U$. It follows that we have weak equivalences

$$
\pi_{0}\left(U \times_{Y} U \times_{\mathcal{M}}^{h} X_{o}\right) \stackrel{p r_{i}^{*} \bar{\alpha}}{\longrightarrow} p r_{i}^{*} V
$$

and so we obtain an isomorphism over $U \times_{Y} U$,

$$
\left[\left(p r_{2}^{*} \bar{\alpha}\right)^{-1} \circ p r_{1}^{*} \bar{\alpha}\right]: p r_{1}^{*} V \rightarrow p r_{2}^{*} V .
$$

The simplicial identities imply that this isomorphism satisfies the hypotheses for descent for morphisms, which implies that there exists $V^{\prime} \rightarrow Y \in \mathcal{C}$ together with an isomorphism $V \cong V^{\prime} \times_{Y} U$ making the following diagram commute.

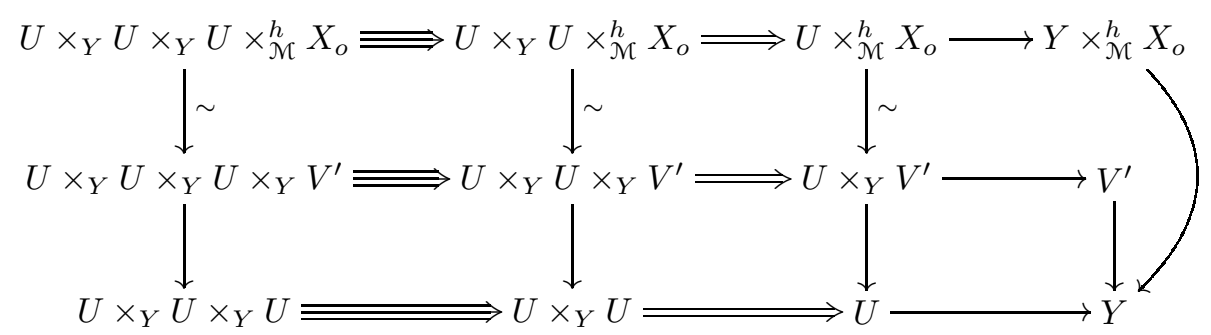

It follows that $V^{\prime}$ is weakly equivalent to

$$
\left|U_{\bullet} \times_{Y} V^{\prime}\right| \stackrel{\sim}{\longleftarrow}\left|U_{\bullet} \times \times_{\mathcal{M}} X_{o}\right| \stackrel{\sim}{\longrightarrow} Y \times_{\mathcal{M}}^{h} X_{o} .
$$

Essentially the same argument implies that the diagonal $\mathcal{M} \rightarrow \mathcal{M} \times \mathcal{M}$ is representable. 
To see that $X_{o} \rightarrow \mathcal{M}$ is a cover, consider the pullback square

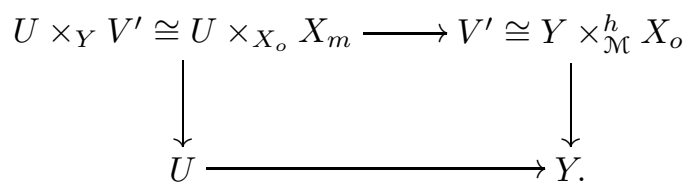

The bottom horizontal arrow and left vertical one are covers since $X_{m} \rightarrow X_{o}$ is a cover. Since the topology on $\mathcal{C}$ is local, $V^{\prime} \rightarrow Y$ is a cover.

By [SGA, Exposé IX], we have the following corollary.

Corollary 4.6. Let $\mathcal{C}=$ Schemes in the étale topology. Then $\mathcal{M}$ is a DeligneMumford stack if and only if it is weakly equivalent to a groupoid object $\left(X_{o}, X_{m}\right)$ in $\mathrm{C}$, with $X_{m} \rightarrow X_{o}$ a cover, and the diagonal of $\mathcal{N}$ is quasicompact and separated.

The flat topology on affine schemes satisfies descent for morphisms by [SGA, Theorem VIII.2.1], so we have the following corollary.

Corollary 4.7. Let $\mathrm{C}=$ Affine schemes in the flat topology. The generalized algebraic stacks are those stacks which are weakly equivalent to flat Hopf algebroids.

\section{Appendix A. TOpologies on A STACK}

We define two new sites $\operatorname{Rep} / \mathcal{M}$ and $P(\mathcal{C}, \mathcal{G r p d}) / \mathcal{M}$ associated to a presheaf of groupoids $\mathcal{M}$. We show that if $\mathcal{M}$ is a generalized algebraic stack, then the category of sheaves $S h(\operatorname{Rep} / \mathcal{M})$ agrees with the usual category of sheaves on $\mathcal{M}$. In addition we prove a comparison theorem explaining the relation between $\operatorname{Rep} / \mathcal{M}$ and $P(\mathcal{C}, \mathcal{G r p d}) / \mathcal{M}$.

A.1. The site $\mathcal{C} / \mathcal{M}$. In this section we recall a site canonically associated to a presheaf of groupoids $\mathcal{M}$ first considered in [DM, Definition 4.10].

Definition A.1. Let $\mathcal{M}$ be in $P(\mathcal{C}, \mathcal{G r p d})$ and let $\mathcal{C} / \mathcal{M}$ denote the site whose

- objects are pairs $(X, f)$, where $X \in \mathcal{C}$ and $X \stackrel{f}{\longrightarrow} \mathcal{M}$,

- morphisms from $X \stackrel{f}{\longrightarrow} \mathcal{M}$ to $X^{\prime} \stackrel{g}{\longrightarrow} \mathcal{M}$ are pairs $(h, \alpha)$, where $X \stackrel{h}{\longrightarrow} X^{\prime}$ and $\alpha$ is a homotopy $f \rightarrow g \circ h$,

- covers are collections of morphisms which forget to covers in $\mathcal{C}$.

For a proof that this defines a Grothendieck topology, see [H2, Section 2.1].

Remark A.2. Given maps $f, f^{\prime}: X \rightarrow \mathcal{M}$, a homotopy $\alpha: f \rightarrow f^{\prime}$ determines an isomorphism in $\mathcal{C} / \mathcal{M}$ between the objects $f$ and $f^{\prime}$. So a presheaf $F$ on $\mathcal{C} / \mathcal{M}$ will satisfy $F(X, f) \cong F\left(X, f^{\prime}\right)$. The category $\mathcal{C} / \mathcal{M}$ is just the Grothendieck construction on the functor $\mathcal{N}$.

Remark A.3. Definition A.1 generalizes the étale site [DM, 4.10] of a DeligneMumford stack, which is the site defined above for $\mathcal{C}$ the category of schemes and étale maps in the étale topology. However, there is no site $\mathcal{C}$ which gives rise via A.1 to the smooth-étale site [LM-B, 12.1] of an algebraic stack $\mathcal{M}$. For example, if we take $\mathcal{M}$ to be a scheme, the smooth-étale site is not the over category of $\mathcal{M}$ in some category of schemes which are the only kind of sites which arise via A.1. 
A.2. The site $\operatorname{Rep} / \mathcal{M}$. The concept of representable morphism allows us to extend in a natural way the notion of cover to presheaves of groupoids and so gives rise to the following site.

Definition A.4. For $\mathcal{M}$ in $P(\mathcal{C}, \mathcal{G r p d})$ the site $\operatorname{Rep} / \mathcal{M}$ has

- Objects: strongly representable morphisms $\mathcal{N} \rightarrow \mathcal{M}$,

- Morphisms from $\mathcal{N}_{1} \stackrel{f_{1}}{\longrightarrow} \mathcal{M}$ to $\mathcal{N}_{2} \stackrel{f_{2}}{\longrightarrow} \mathcal{M}$ that consist of pairs $(g, \alpha)$ with $g: \mathcal{N}_{1} \rightarrow \mathcal{N}_{2}$ and $\alpha$ a homotopy $f_{1} \rightarrow f_{2} \circ g$,

- Covers: collections of morphisms $\left\{\mathcal{N}_{i} \stackrel{u_{i}}{\longrightarrow} \mathcal{N}\right\}$ such that the $u_{i}$ are strongly representable and for each $X \rightarrow \mathcal{N}, X \in \mathcal{C}$ the collection $\left\{X \times{ }_{\mathcal{N}}^{h} \mathcal{N}_{i} \rightarrow X\right\}$ is weakly equivalent to a cover in $\mathcal{C}$.

Note that the pullback in $R e p / \mathcal{M}$ is exactly the homotopy fiber product of Definition 2.1. It is also true that homotopy equivalences are isomorphisms in $\operatorname{Rep} / \mathcal{M}$. The proof that $\operatorname{Rep} / \mathcal{M}$ is a site is parallel to that for $\mathrm{C} / \mathcal{M}$.

Proposition A.5. Let $\mathcal{M}$ be a generalized algebraic stack. Then the category of sheaves on $\operatorname{Rep} / \mathcal{M}$ is equivalent to the category of sheaves on the site $\mathcal{C} / \mathcal{M}$.

Proof. Since $\mathcal{M}$ is a generalized algebraic stack, $\mathcal{C} / \mathcal{M}$ embeds in $\operatorname{Rep} / \mathcal{M}$ as a full subcategory. By [Ta, Proposition 3.9.1] it is enough to see that any object in $R e p / \mathcal{M}$ is covered by an object in $\mathrm{C} / \mathcal{M}$.

Given an object $f: \mathcal{N} \rightarrow \mathcal{M}$ in $\operatorname{Rep} / \mathcal{M}$, and a cover $X \rightarrow \mathcal{M}$ with $X \in \mathcal{C}, X \times{ }_{\mathcal{M}}^{h} \mathcal{N}$ is levelwise weakly equivalent to a representable $Z \cong \pi_{0}\left(X \times_{\mathcal{M}}^{h} \mathcal{N}\right)$. The quotient map $p: X \times_{\mathcal{M}}^{h} \mathcal{N} \rightarrow Z$ is a trivial fibration and $Z$ is cofibrant, so $p$ is a homotopy equivalence and hence an isomorphism in $\operatorname{Rep} / \mathcal{M}$. It follows that $Z \rightarrow X \times{ }_{\mathcal{M}}^{h} \mathcal{N} \rightarrow \mathcal{N}$ is a cover in $\operatorname{Rep} / \mathcal{M}$.

Note A.6. One can make a definition analogous to Definition A.4 using the concept of representable morphism instead of strongly representable morphism, but then the result of the previous proposition would not hold as local weak equivalences would not be isomorphisms in the category.

Remark A.7. Let $\left\{f_{i}: \mathcal{U}_{i} \rightarrow \mathcal{N}\right\}$ be a collection of representable morphisms and $\left\{\tilde{f}_{i}: \tilde{U}_{i} \rightarrow \mathcal{N}\right\}$ be the family of fibrations obtained by functorial factorization in $P(\mathcal{C}, \mathcal{G r p d})_{L}$. Then the following are equivalent:

(i) the collection $\left\{f_{i}: \mathcal{U}_{i} \rightarrow \mathcal{N}\right\}$ is a cover in the sense of Definition 3.2;

(ii) the collection $\left\{\tilde{f}_{i}: \tilde{U}_{i} \rightarrow \mathcal{N}\right\}$ is a cover in $\operatorname{Rep} / \mathcal{N}$.

A.3. The site $P(\mathcal{C}, \mathcal{G r p d}) / \mathcal{M}$. We now define a site associated to $\mathcal{M}$, which is very natural from the point of view of homotopy theory, and compare it to the ones discussed above.

Theorem A.8. Let $\mathcal{C}$ be a site and $\mathcal{N} \in P(\mathcal{C}, \mathcal{G r p d})_{L}$. Then there is a Grothendieck topology on $P(\mathcal{C}, \mathcal{G r p d})_{L} / \mathcal{N}$ in which the covers are the sets of morphisms $\left\{\mathcal{U}_{i} \rightarrow \mathcal{N}\right\}$ that satisfy the following:

- $\mathcal{U}_{i} \rightarrow \mathcal{N}$ are fibrations,

- $\left|\mathcal{U}_{\bullet}\right| \rightarrow \mathcal{N}$ is a weak equivalence.

Proof. First we prove that pullbacks of covers are covers. Let $\coprod \mathcal{U}_{i} \rightarrow \mathcal{N}$ be a cover and $\mathcal{M} \rightarrow \mathcal{N}$ a morphism. The morphism $\amalg \mathcal{U}_{i} \rightarrow \mathcal{N}$ is an objectwise fibration and so the induced map $\left|\mathcal{U}_{\bullet}\right| \rightarrow \mathcal{N}$ is also an objectwise fibration. As geometric 
realization commutes with fiber products, $\left|\mathcal{U}_{\bullet}\right| \times_{\mathcal{N}} \mathcal{M} \cong\left|\mathcal{U}_{\bullet} \times_{\mathcal{N}} \mathcal{M}\right|$ and so we have a pullback square

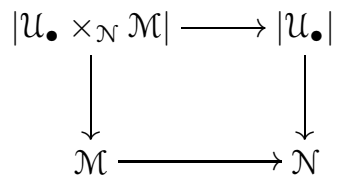

where the right vertical map is an objectwise fibration and a weak equivalence. By $[\mathrm{H}$, Corollary 5.8] the pullback of a weak equivalence that is an objectwise fibration is a weak equivalence.

To see that covers compose, let $\left\{\mathcal{V}_{i j} \rightarrow \mathcal{U}_{i}\right\}$ be covers of each $\mathcal{U}_{i}$. The iterated fiber products of the covers $\left\{\mathcal{V}_{i j} \rightarrow \mathcal{U}_{i}\right\}$ form a bisimplicial object $\mathcal{V}_{\bullet, \bullet}$ augmented over $\mathcal{U}_{\bullet}$. The columns $\mathcal{V}_{n, \bullet}$ are iterated fiber products of the nerves of $\left\{\mathcal{V}_{i j} \rightarrow \mathcal{U}_{i}\right\}$, and therefore the map induced by the augmentation

$$
\left|\mathcal{V}_{\bullet, \bullet}\right| \rightarrow\left|\mathcal{U}_{\bullet}\right| \rightarrow \mathcal{N}
$$

is a weak equivalence. The geometric realization of the bisimplicial object is equivalent to the geometric realization of its diagonal, so $\left|\operatorname{diag} \mathcal{V}_{\bullet, \bullet}\right| \rightarrow \mathcal{N}$ is a weak equivalence.

The nerve of the cover $\left\{\mathcal{V}_{i j} \rightarrow \mathcal{N}\right\}$ is the 0 -th row $\mathcal{V}_{\bullet, 0}$. Since $\mathcal{V}_{\bullet, 0}$ is a 0 coskeleton over $\mathcal{N}$, there is a retraction to the canonical map $\mathcal{V}_{\bullet, 0} \rightarrow \operatorname{diag} \mathcal{V}_{\bullet}, \bullet$ over $\mathcal{N}$ (see [DHI, Proposition A4]) and therefore $\mathcal{V}_{\bullet, 0} \rightarrow \mathcal{N}$ is a weak equivalence.

Proposition A.9. Given a collection $\left\{f_{i}: \mathcal{U}_{i} \rightarrow \mathcal{N}\right\}$ in $P(\mathcal{C}, \mathcal{G r p d}) / \mathcal{N}$ the following are equivalent:

(i) The collection $\left\{f_{i}: \mathcal{U}_{i} \rightarrow \mathcal{N}\right\}$ is a cover in $P(\mathcal{C}, \mathcal{G r p d}) / \mathcal{M}$.

(ii) For each $X \rightarrow \mathcal{N}$ the collection $\left\{\mathcal{U}_{i} \times_{\mathcal{N}} X \rightarrow X\right\}$ is a cover in $P(\mathcal{C}, \mathcal{G r p d}) / \mathcal{M}$.

(iii) For each $X \rightarrow \mathcal{N}$ the union of the images of $\mathfrak{U}_{i} \times \mathcal{N} X \rightarrow X$ is a covering sieve of $X$ in $\mathrm{C}$.

Proof. The fact that (i) implies (ii) is a part of the axioms for a topology. First we prove that (ii) implies (i). Given $X \in \mathcal{C}$, and $X \rightarrow \mathcal{N}$, let $W$ denote $\left|\mathcal{U}_{\bullet}\right|$. Then the projection map $W \times \mathcal{N} X \rightarrow X$ is a weak equivalence since

$$
W \times_{\mathcal{N}} X \cong\left|\mathcal{U}_{\bullet} \times_{\mathcal{N}} X\right| \cong\left|\left(\mathcal{U} \times_{\mathcal{N}} X\right) \bullet\right|,
$$

so this map is the induced map to $X$ from the nerve of the cover $\mathfrak{U}_{i} \times_{\mathcal{N}} X \rightarrow X$.

Similarly, given any map $X \otimes \Delta^{1} \rightarrow \mathcal{N}$, the pullback $\left(X \otimes \Delta^{1}\right) \times_{\mathcal{N}} W \rightarrow X \otimes \Delta^{1}$ is a weak equivalence since in the diagram

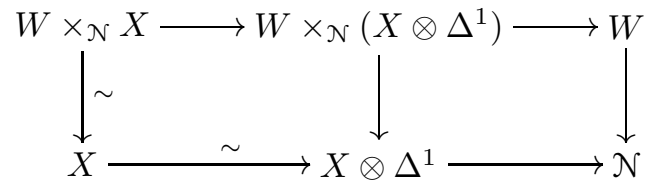

the top left map is a levelwise weak equivalence (because $W \rightarrow \mathcal{N}$ is a levelwise fibration and $\mathcal{G r p d}$ is right proper). It is now straightforward to check that $W \rightarrow \mathcal{N}$ is a weak equivalence using the local lifting conditions (Definition 1.2).

To see that (ii) implies (iii) let $\left\{\mathcal{U}_{i} \rightarrow X\right\}$ be a cover in $P(\mathcal{C}, \mathcal{G r p d}) / \mathcal{M}$ and let $F$ be any sheaf on $\mathcal{C}$. $F$ is a discrete stack and so

$$
\operatorname{Map}(X, F) \stackrel{\sim}{\longrightarrow} \operatorname{holim} \operatorname{Map}\left(\mathcal{U}_{\bullet}, F\right) \cong \lim \operatorname{Map}\left(\mathcal{U}_{\bullet}, F\right) \cong \operatorname{Map}\left(\operatorname{colim} \pi_{0} \mathcal{U}_{\bullet}, F\right),
$$


which shows that $X$ is the coequalizer of the sheafification $\left(\coprod \pi_{0} \mathcal{U}_{i j} \rightrightarrows \coprod \pi_{0} \mathcal{U}_{i}\right)$, so by [MM, Corollary III.7.7] the union of the images of $\pi_{0} \mathcal{U}_{i} \rightarrow X$ is a covering sieve in $\mathrm{C}$.

Conversely suppose that $\left\{\mathcal{U}_{i} \rightarrow X\right\}$ generates a covering sieve. This means that $\operatorname{sh}\left(\coprod \pi_{0} \mathcal{U}_{i}\right) \rightarrow X$ is a surjection of sheaves, from which it follows that colim $\pi_{0} \mathcal{U}_{\bullet} \rightarrow$ $X$ is a weak equivalence. Since $\mathcal{U}_{\bullet}$ is a 0 -coskeleton in simplicial objects over $X$, the projection $\left|\mathcal{U}_{\bullet}\right| \rightarrow \operatorname{colim} \pi_{0} \mathcal{U}_{\bullet}$ is a levelwise weak equivalence. It follows that $\left\{\mathcal{U}_{i} \rightarrow X\right\}$ is a cover in $P(\mathcal{C}, \mathcal{G r p d}) / \mathcal{M}$.

Here is the relation between the notion of cover on $P(\mathcal{C}, \mathcal{G r p d}) / \mathcal{M}$ just defined with the ones defined previously.

Corollary A.10. Let $\left\{f_{i}: \mathcal{U}_{i} \rightarrow \mathcal{N}\right\}$ be a collection of representable morphisms and $\left\{\tilde{f}_{i}: \tilde{U}_{i} \rightarrow \mathcal{N}\right\}$ be fibrations obtained by functorial factorization in $P(\mathcal{C}, \mathcal{G r p d})_{L}$.

(1) If $\left\{f_{i}: \mathcal{U}_{i} \rightarrow \mathcal{N}\right\}$ is a cover in the sense of Definition 3.2 , then $\left\{\tilde{f}_{i}: \tilde{U}_{i} \rightarrow \mathcal{N}\right\}$ is a cover in $P(\mathcal{C}, \mathcal{G r p d}) / \mathcal{M}$.

(2) Conversely, if $\left\{\tilde{f}_{i}: \tilde{U}_{i} \rightarrow \mathcal{N}\right\}$ is a cover in $P(\mathcal{C}, \mathcal{G r p d}) / \mathcal{M}$, then for each $X \rightarrow \mathcal{N}$ the collection $\left\{\mathcal{U}_{i} \times{ }_{\mathcal{N}}^{h} X \rightarrow X\right\}$ determines a covering sieve in $\mathcal{C}$.

Let $S h(\mathcal{M})$ be the category of sheaves on $P(\mathcal{C}, \mathcal{G r p d})_{L} / \mathcal{N}$ that take weak equivalences to isomorphisms. The above corollary implies that we have a surjective restriction functor $S h(\mathcal{M}) \rightarrow S h(\mathcal{C} / \mathcal{M})$.

\section{REFERENCES}

Bry. J.-L. Brylinski, Loop Spaces, Characteristic Classes, and Geometric Quantization, Progress in Mathematics 107, Birkhaüser, Basel, 1993. MR1197353 (94b:57030)

DM. P. Deligne and D. Mumford, The irreducibility of the space of curves of given genus, Inst. Hautes Études Sci. Publ. Math. 36 (1969), 75-109. MR0262240 (41:6850)

DHI. D. Dugger, S. Hollander and D. Isaksen, Hypercovers and simplicial presheaves, Math. Proc. Cambridge Philos. Soc. 136 (2004), no. 1, 9-51. MR2034012 (2004k:18007)

SGA. A. Grothendieck, Revêtements étales et groupe fondamental, Séminaire de Géométrie Algébrique du Bois-Marie 1960-1961 (SGA 1), Lecture Notes in Mathematics, Vol. 224, Springer-Verlag, Berlin-New York, 1971. MR0354651 (50:7129)

G. P. Goerss, (Pre-)Sheaves of Ring Spectra on the Moduli Stack of Formal Group Laws, 101131, NATO Sci. Ser. II Math. Phys. Chem., 131, Kluwer Acad. Publ., Dordrecht, 2004. MR2061853 (2005d:55007)

Gi. J. Giraud, Cohomologie non-abelienne, Springer-Verlag, Berlin, Heidelberg, New York, 1971. MR0344253 (49:8992)

H. S. Hollander, A Homotopy Theory for Stacks, math.AT/0110247

H2. S. Hollander, Descent for quasi-coherent sheaves on stacks, preprint (2006).

LM-B. G. Laumon, L. Moret-Bailly, Champs Algébriques, Ergeb. der Math, Vol. 39, SpringerVerlag, Berlin, 2000. MR1771927 (2001f:14006)

MM. S. Mac Lane and I. Moerdijk, Sheaves in Geometry and Logic: A First Introduction to Topos Theory, Springer-Verlag, Berlin, Heidelberg, New York, 1992. MR1300636 (96c:03119)

Mi. J. Milne, Étale cohomology, Princeton Mathematical Series 33, Princeton University Press 1980, 323 pp. MR0559531 (81j:14002)

P. E. Pribble, Algebraic Stacks for Stable Homotopy Theory and the Algebraic Chromatic Convergence Theorem, Northwestern University Thesis, 2004.

Ta. G. Tamme, Introduction to étale cohomology. Universitext. Springer-Verlag, Berlin, 1994. x+186 pp. MR1317816 (95k:14033) 
Department of Mathematics, Hebrew University, Jerusalem, Israel

E-mail address: sjh@math.huji.ac.il+

Current address: Centro de Análise Mathematica, Geometria e Sistemas Dinâmicos, Instituto Superior Técnico, Tech. Univ. Lisbon, Portugal

E-mail address: sjh@math.ist.utl.pt 